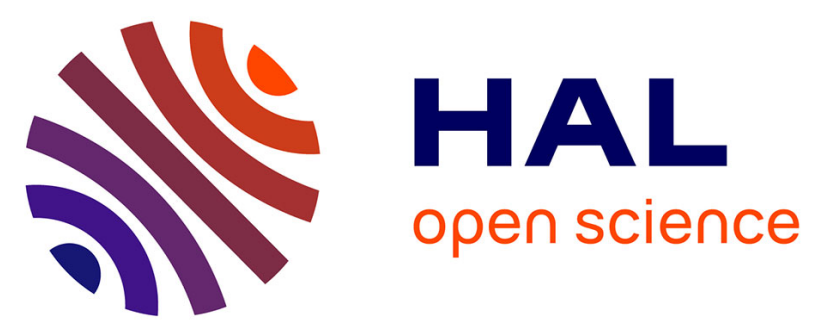

\title{
Sécurisation de la prise en charge médicamenteuse des patients sous antipsychotiques : impact de la collaboration médico-pharmaceutique à la Maison d'Arrêt de Lyon-Corbas
}

C. Picard, L. Lalande, C. Bertin, C. Abel-Coindoz, A. Mauchauffée, G. Giret, L. Zimmer, D. Cabelguenne

\section{To cite this version:}

C. Picard, L. Lalande, C. Bertin, C. Abel-Coindoz, A. Mauchauffée, et al.. Sécurisation de la prise en charge médicamenteuse des patients sous antipsychotiques: impact de la collaboration médico-pharmaceutique à la Maison d'Arrêt de Lyon-Corbas. L'Encéphale, 2019, 45, pp.482 - 487. 10.1016/j.encep.2019.05.008 . hal-03488422

\section{HAL Id: hal-03488422 \\ https://hal.science/hal-03488422}

Submitted on 21 Dec 2021

HAL is a multi-disciplinary open access archive for the deposit and dissemination of scientific research documents, whether they are published or not. The documents may come from teaching and research institutions in France or abroad, or from public or private research centers.
L'archive ouverte pluridisciplinaire HAL, est destinée au dépôt et à la diffusion de documents scientifiques de niveau recherche, publiés ou non, émanant des établissements d'enseignement et de recherche français ou étrangers, des laboratoires publics ou privés.

\section{(c) (1) $\$$}

Distributed under a Creative Commons Attribution - NonCommerciall 4.0 International 


\section{Sécurisation de la prise en charge médicamenteuse des patients sous antipsychotiques : impact de la collaboration médico-pharmaceutique à la Maison d'Arrêt de Lyon-Corbas}

Ensuring safety of patients under antipsychotic drugs: Impact of medical and pharmaceutical collaboration at prison.

\section{Camille Picard ${ }^{1 *}$, Laure Lalande ${ }^{1}$, Camille Bertin ${ }^{1}$, Chloé Abel-coindoz ${ }^{1}$, Alice

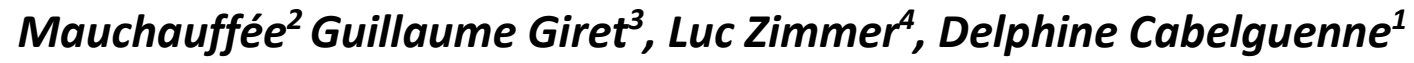

${ }^{1}$ Unité de Pharmacie Clinique en milieu carcéral, Groupement Hospitalier Sud, Hospices Civils de Lyon, Lyon, France

${ }^{2}$ Unité sanitaire en milieu pénitentiaire, Groupement Hospitalier Sud, Hospices Civils de Lyon, Lyon, France

${ }^{3}$ Pôle santé mentale des détenus et psychiatrie légale, Centre Hospitalier du Vinatier, Bron, France

${ }^{4}$ CERMEP, Groupement Hospitalier Est, Université Claude Bernard Lyon1, Hospices Civils de Lyon, INSERM, CNRS, Lyon, France

Pas de conflit d'intérêt.

* Pour correspondance : Camille Picard

Maison d'Arrêt Lyon-Corbas, Unité sanitaire, service Pharmacie, 40 Boulevard des Nations, 69960 Corbas

Email : camille.picard@chu-lyon.fr 


\section{Sécurisation de la prise en charge médicamenteuse des patients sous antipsychotiques : impact de la collaboration médico-pharmaceutique à la Maison d'Arrêt de Lyon-Corbas}

\section{Ensuring safety of patients under antipsychotic drugs: Impact of medical and pharmaceutical collaboration at prison.}

\section{Résumé}

Objectif : L'iatrogénie des antipsychotiques, en plus des facteurs liés au mode de vie, est en partie responsable de la surmortalité des patients traités. Un suivi clinique et paraclinique des patients est recommandé et nécessaire. Dans ce contexte un projet collaboratif a été mené au sein de l'unité sanitaire sur l'optimisation du suivi des patients sous antipsychotiques dans le contexte du milieu pénitentiaire. Sont associés dans ce projet médecins généralistes, psychiatres et pharmaciens. L'objectif de l'étude est d'évaluer l'évolution de la qualité du suivi des patients traités par antipsychotiques. Méthode : Il s'agit d'une étude rétrospective incluant tous les patients suivis et traités par antipsychotiques pendant au moins 6 mois durant les années 2011 et 2015. Le recueil de données a été réalisé à partir des dossiers médicaux. L'indicateur choisi pour apprécier la qualité du suivi était le pourcentage de conformité des paramètres paracliniques pour chacun des patients au regard du référentiel prédéfini en concertation médico-pharmaceutique. Résultats : En 2015 versus 2011, la qualité de suivi s'est améliorée pour 9 des 10 paramètres étudiés, avec une amélioration statistiquement significative pour 7 d'entre eux : Indice de Masse Corporelle, bilan lipidique, numération formule sanguine, bilan hépatique, ionogramme, glycémie et fonction rénale. Conclusion : Cette étude a permis de démontrer que les axes d'amélioration définis en concertation médico-pharmaceutique ont eu un impact effectif sur la qualité de suivi des patients sous antipsychotiques.

Mots clés : antipsychotique, suivi clinico-biologique, prison

\section{Abstract}

Objectives: Antipsychotics are the standard treatment for psychosis. However, when combined with other lifestyle factors they are partially responsible for an excessive mortality rate. A clinical and paraclinical monitoring of patients is therefore necessary. In 2011, this element led doctors and pharmacists to improve monitoring and formalize a follow-up adapted to inmate patients. The aim of this study was to assess the impact of medicalpharmaceutical collaboration on monitoring quality of patients treated by antipsychotics. 
Methods: This is a retrospective study including all patients treated by antipsychotics for at least 6 months in 2011 and again in 2015. Data were collected from medical files. The indicator assessing the monitoring quality was the compliance percentage, of registred parameters for each patient on the basis of specific guidelines. Results: In 2015 compared to 2011, the monitoring quality increased for 9 out of 10 parameters. This improvement was statisticaly significant for 7 of them : Body Mass Index, lipid test, complete blood count, transaminase, ionogram, glycemia, glomerular filtration rate. Conclusion: The actions of improvement collectivelly implemented in 2011 had a concrete impact on patients in the follow-up in 2015.

\section{Key-words : antipsychotic, clinical and biological follow-up, prison}

\section{Introduction}

Le taux de mortalité des patients schizophrènes est 3 à 4 fois supérieur à celui de la population générale $(1,2)$ avec une espérance de vie diminuée, en moyenne, de $20 \%(2,3)$. Les maladies cardiovasculaires sont à présent la première cause de cette surmortalité devant le suicide (4). Les comorbidités cardiovasculaires et métaboliques sont liées d'une part au mode de vie de ces patients (sédentarité, tabagisme, régime alimentaire, etc.) et d'autre part à l'iatrogénie des traitements antipsychotiques (AP). Les effets métaboliques des AP regroupent des diabètes de type 2 , des dyslipidémies et des prises de poids importantes. Tous les AP sont susceptibles d'induire une prise de poids mais cet effet est plus marqué avec les AP de seconde génération (5). Pour exemple, l'olanzapine entraîne une prise de poids supérieure à $7 \%$ du poids initial chez $40 \%$ des patients (6). La prise de poids, en plus d'être un facteur de risque métabolique et cardiovasculaire, est également un important facteur de non-adhésion aux traitements médicamenteux. D'après le réseau des Centres Experts Schizophrénie, la prévalence du syndrome métabolique est de $24,2 \%$ soit deux fois plus que dans la population générale et ne serait majoritairement pas correctement prise en charge (4). Concernant le diabète, son incidence serait deux à quatre fois plus élevée chez les patients schizophrènes y compris chez les patients non traités (6). Ces patients seraient plus vulnérables à cette pathologie, la prise d'AP pouvant déclencher ou aggraver ce diabète. Les causes d'apparition de cette maladie restent complexes et ne sont pas seulement liées aux facteurs de risques classiques telles que la prise de poids, ni la dose d’AP utilisée $(7,8)$.

Les AP peuvent être à l'origine de troubles de la dépolarisation, se traduisant par un allongement de l'espace QT et augmentant le risque de survenue de troubles du rythme 
ventriculaire tels que les torsades de pointe (TdP). En effet, la prise régulière d'AP majorerait d'un facteur 3 le risque de mort subite (9). Ce risque augmente en cas d'association à d'autres médicaments dits torsadogènes (autres AP, méthadone, hydroxyzine, escitalopram ou venlafaxine par exemple) et en cas d'hypokaliémie (10).

Afin de prévenir la survenue de ces effets indésirables, un suivi clinique et paraclinique des patients au long cours est recommandé. Ce suivi est actuellement formalisé par des recommandations nationales et internationales (11-14).

\section{Contexte}

En 2018 à la Maison d'arrêt (MA), sur 887 détenus présents, 293 (46 \%) bénéficiaient d'un traitement médicamenteux au long cours et parmi eux 242 (60\%) avaient un traitement à visée psychiatrique (troubles du sommeil et anxiété compris).

Au sein de l'unité sanitaire de la MA, la vérification de l'exhaustivité du suivi paraclinique des patients sous traitements AP se fait à l'occasion de l'analyse systématique de toutes les prescriptions médicamenteuses par le pharmacien (analyse de niveau 3 selon la classification de la Société Française de Pharmacie Clinique) (15). Cette analyse peut donner lieu, entre autre, à des demandes de suivi thérapeutique auprès du clinicien référent.

Par ailleurs, des réunions de concertation médico-pharmaceutique (MP) sont organisées tous les mois depuis 2001. En 2012, dans le cadre de ces réunions, un état des lieux de la qualité du suivi des patients sous AP a été réalisé. II a montré que pour les différents paramètres paracliniques, la qualité de leur suivi était qualifiée de modérée à insuffisante. Suite à cet état des lieux, il a été décidé de retenir, comme action d'évaluation des pratiques professionnelles (EPP), l'optimisation du suivi des patients sous AP et la prévention du risque iatrogène lié à cette classe médicamenteuse. Ont été adoptées comme référentiels les recommandations nationales et internationales (11-14). Ces recommandations ont été appliquées dans un premier temps, puis nous les avons aménagées afin de les adapter au contexte carcéral. Cette adaptation était nécessaire en raison des difficultés de « mouvements » en détention (le patient est enfermé dans sa cellule et ne dispose pas des clés pour en sortir). Les patients peuvent être également en état d'agitation ou d'agressivité, 
situations peu propices à proposer un prélèvement sanguin. Une fiche de suivi a alors été élaborée et mise en place en 2013. Elle est classée dans le dossier médical du patient (fig.1).

\section{Objectif}

L'objectif de cette étude était d'évaluer l'impact de la mise en place de l'action d'EPP " optimisation du suivi paracliniques des patients sous AP » en comparant la qualité du suivi des patients traités par AP à la MA pour l'année 2015 versus 2011.

\section{Patients et méthodes}

\section{Critères d'inclusion et d'exclusion}

II s'agit d'une étude rétrospective dans laquelle ont été inclus tous les patients traités par AP pendant au moins 6 mois, que le traitement ait été instauré ou non au cours de la détention. Le recueil de données de 2011 concernait uniquement les patients pour lesquels les diagnostics F20 à F29 du CIM-10 ont été retenus (schizophrénie, troubles schizotypiques et troubles délirants notamment). Pour le recueil de l'année 2015, nous avons choisi d'inclure tous les patients traités par AP quel que soit le diagnostic. Les patients bénéficiant d'un traitement AP à visée uniquement sédative sur plusieurs mois devaient également avoir un suivi régulier des potentiels effets indésirables.

Les patients dont le refus de soin, et en particulier le refus des prélèvements biologiques, ont été signalés dans le dossier médical a au moins 2 reprises n’ont pas été comptabilisés. En effet, nous avons considéré que pour ces patients, le défaut de suivi thérapeutique n'était pas du fait du clinicien qui avait prescrit le bilan mais plutôt du fait du patient qui avait refusé la convocation à l'unité sanitaire.

\section{$\underline{\text { Recueil de données }}$}

Le recueil de données a été fait rétrospectivement à partir des dossiers médicaux des patients. Pour chaque patient, plusieurs séries de paramètres paracliniques pouvaient être recueillies à des temps différents.

En plus de l'âge et du sexe, les données recueillies dans le contexte de ce suivi ont été les suivantes:poids, taille, indice de masse corporelle (IMC), tension artérielle (TA), 
électrocardiogramme (ECG), ionogramme, numération formule sanguine (NFS), bilan hépatique $(B H)$, bilan lipidique $(B L)$, glycémie et débit de filtration glomérulaire (DFG).

\section{Traitement et analyse des données}

Pour chaque paramètre suivi, un pourcentage de conformité (PC) a été calculé. II s'agit du rapport entre le nombre de mesures effectuées pour le paramètre suivi et le nombre attendu de fois où ce paramètre aurait dû être mesuré. À chaque résultat de $\mathrm{PC}$ correspond une qualité de suivi : insuffisant ( $P C<25 \%)$, modéré $(25 \% \leq P C<50 \%)$, satisfaisant $(50 \% \leq P C<75 \%)$ et excellent ( $P C \geq 75 \%)$.

Afin d'explorer les facteurs extérieurs qui ont pu influencer le suivi des patients, huit sousgroupes ont été distingués selon :

- La durée de détention ( $<1$ an, de 1 à 2 ans, $>2$ ans).

- La valeur de I'IMC $(<25, \geq 25)$.

- La présence ou non de facteurs de risque cardiovasculaire associés : diabète, dyslipidémie, obésité, hypertension, antécédents cardiovasculaires.

- La co-prescription ou non d'au moins un médicament à risque de toxicité cardiaque.

- La prescription ou non d'un autre AP.

- La prescription ou non d'une forme retard.

- Le type de prise en charge : ambulatoire pour les patients consultant à l'unité sanitaire ou en hôpital de jour (unité d’hébergement).

- L'utilisation ou non du traitement AP uniquement à visée sédative.

Le traitement des données a été réalisé à l'aide du logiciel IBM SPSS Statistics 19. Toutes les comparaisons d'effectifs ont été réalisées à l'aide du test du Khi-2. Pour chaque test, le seuil de significativité a été défini pour une p-value strictement inférieure à 0.01 .

\section{Résultats}


Cette étude a inclus 121 patients (61 pour la cohorte 2011 et 60 pour la cohorte 2015). Parmi eux, on comptait 13 femmes pour 108 hommes, reflet du sex-ratio des détenus de la MA (690 places d'hommes pour 60 places de femmes).

Dans la cohorte de 2011, la pression artérielle était le seul paramètre avec un PC moyen supérieur à $50 \%$. En 2015, c'est le cas de quatre autres paramètres : I'hémogramme, les transaminases, l'ionogramme et le débit de filtration glomérulaire. D'autres paramètres sont en amélioration : I'IMC, la glycémie, le bilan lipidique et l'ECG. La qualité de suivi du poids a été stable mais toujours inférieure à 50 \% entre 2011 et 2015 (cf. tableau 1).

Pour l'ensemble des paramètres, à l'exception de la pression artérielle, la qualité de suivi était significativement supérieure en 2015 versus 2011, avec une augmentation des effectifs de patients ayant des suivis classés satisfaisant à excellent pour la plupart des paramètres. Seuls le suivi du poids et le contrôle des ECG n'atteignent pas la significativité (cf. figure 2).

L'analyse par sous-groupe a mis en évidence que la durée du séjour, l'IMC du patient, la présence de facteurs de risque cardiovasculaire, la co-prescription de plusieurs AP ou d'autres médicaments à risque de torsade de pointe ne semblent pas avoir d'impact sur le suivi paraclinique des patients. En revanche, le fait d'être admis en hôpital de jour semble améliorer le suivi biologique des patients avec en particulier une différence statistiquement significative pour le suivi d'ionogramme $(p=0,003)$ et de la fonction rénale $(p=0,008)$. La prescription de formes injectables semble également améliorer le suivi de l'ECG et des paramètres biologiques (différences non significatives). Enfin, la prescription d'AP à visée uniquement sédative semble corrélée à une moins bonne qualité de suivi pour le bilan lipidique $(p=0,008)$, la NFS $(p=0,001)$, l'ionogramme $(p<0,001)$, la glycémie $(p=0,001)$, la fonction rénale $(p<0,001)$, les transaminases $(p=0,011)$ et l’ECG $(p=0,04)$.

\section{Discussion}

Les différents effets indésirables des AP justifient la mise en place d'un suivi clinique et biologique chez les patients traités (11-14). Des recommandations nationales et internationales existent mais sont réputées peu suivies (16-18). Notre étude a mis en 
évidence une amélioration de la qualité de ce suivi chez les patients sous AP à la MA en 2015 versus 2011.

Cette amélioration est liée en premier lieu à la décision collégiale en réunion de concertation MP de choisir la prévention des risques de survenue des effets indésirables liés aux AP comme axe fort dans notre démarche d'EPP. L'amélioration du PC moyen des transaminases qui est passé de $36 \%$ à $65 \%$ en est un bon exemple. Le risque de toxicité des AP de type hépatique, cardiovasculaire, hématologique et métabolique a tendance à être sous-estimé par les médecins prescripteurs et impose un suivi paraclinique périodique des patients. La mise en application collégiale des recommandations nationales $(11,12,14)$ de suivi avec adaptation au milieu pénitentiaire (mise en œuvre d'un suivi annuel en l'absence d'anomalie détectée), leur rappel et leur prescription par les cliniciens après analyse des ordonnances par les pharmaciens ont fortement participé à l'amélioration de l'exhaustivité de ce suivi. L'impact positif de la diffusion des recommandations de suivi a été montré dans plusieurs études. Celle de Cotes et collaborateurs en 2015 (18) a montré une amélioration du suivi de la glycémie et du bilan lipidique d'environ $20 \%$ sur 5 des 10 centres inclus dans l'étude. Ces résultats ont été obtenus après des actions de sensibilisation auprès des équipes soignantes sous la forme d'audits et de feed-back au chef de service (18). L'étude de Moeller et collaborateurs en 2011 a aussi montré l'intérêt de la diffusion de bonnes pratiques (19). Dans cette étude monocentrique, dans la première cohorte de 2002-2003, 23 \% des 2204 patients inclus ont eu un dosage de leur glycémie et $10 \%$ ont eu un bilan lipidique. Après la publication et la diffusion des recommandations de I'ADA/APA (American Diabeties Association/American Psychiatric Association) en 2004, ces taux passent respectivement à $75 \%$ et $52 \%$ des 1646 patients inclus entre 2005 et 2007 (19).

Les interventions pharmaceutiques (IP) formulées lors de l'analyse des prescriptions par les pharmaciens sont également un bon vecteur pour le rappel des bonnes pratiques et ont contribué à cette amélioration. Ces IP ont été étudiées sur les 2 périodes de notre étude. Pour l'année 2011, 41 (15,5\%) des 264 IP recensées correspondaient à des demandes de suivi thérapeutique pour des patients sous AP. En 2015, ces demandes s'élevaient à 214 $(14,8 \%)$ sur les 1445 IP formulées auprès des cliniciens. L'augmentation du nombre d'IP entre 2011 et 2015 montre la sensibilisation des pharmaciens à la nécessité du suivi des patients. L'intérêt des pharmaciens pour ce suivi est illustré par l'étude de Parent et al. Au 
cours de cette étude multicentrique, 4620 IP portant sur les psychotropes ont été analysées et il a été observé que sur 112 IP portant sur des demandes de suivi thérapeutique, la quasitotalité (98 IP) concernait des médicaments AP (20). Le rôle du pharmacien dans le suivi des patients sous AP est également soutenu par l'étude de Lee en 2016 (21). Cette étude montre le manque d'efficacité des alertes informatiques lorsqu'elles ne sont pas couplées à l'intervention d'un pharmacien lors de l'analyse des prescriptions médicamenteuses. L'aide des systèmes experts dans l'amélioration du suivi des patients n'est pas à négliger. Dans son étude, Reeves a montré que, couplé à une formation des prescripteurs sur les risques métaboliques liés aux AP, I'ajout d'un programme de suivi biologique au dossier médical électronique des patients a permis d'améliorer leur suivi (22). Dans le cas de notre étude, l'apport de l'outil informatique est modeste, mais il a pourtant permis d'améliorer le suivi de la glycémie chez nos patients. Un nouveau paramétrage de l'outil de prescription propose lors de la prescription d'examens biologiques I'"ionogramme complet" qui comprend, en plus d'ionogramme, le dosage de la créatininémie et de la glycémie à jeun. Il s'agit d'un bon moyen de rappel pour les médecins du suivi périodique de ces paramètres. Ce système pourrait être perfectionné en ajoutant une fonction "Bilan AP », qui présélectionnerait automatiquement tous les examens nécessaires au suivi des patients sous AP.

Mettre en place un système pérenne et efficace de suivi des patients reste complexe (23). Une des principales difficultés est de s'adapter au milieu d'exercice. Cela peut être illustré par le suivi de l'ECG. En effet, dans notre cohorte, ce suivi reste insuffisant, avec un PC moyen de seulement $42 \%$ en 2015 , alors que ce suivi est important dans la prévention de la mort subite. Cela peut s'expliquer par le fait qu'un ECG est plus difficile à réaliser qu'un prélèvement sanguin $d u$ fait de l'organisation des soins. On pourrait penser que l'incarcération facilite l'organisation et la réalisation de cet examen, mais dans les faits, il reste difficile à organiser pour les équipes soignantes. Cette difficulté n'est pas propre au milieu pénitentiaire, elle semble également présente en milieu hospitalier (9). Le problème particulier de la réalisation des ECG a également été étudié par une équipe italienne en 2017. Cette étude montre que la toxicité cardiaque des AP est connue des soignants puisqu'un ECG et un ionogramme étaient demandés plus fréquemment avant l'instauration de AP avec un risque torsadogène connu ou possible, selon la base de données CredibleMeds ${ }^{\circledR}(24)$, par rapport aux AP avec un risque plus faible $(p=0,002)$. Cette différence $n^{\prime}$ est plus significative lorsqu'il s'agit du suivi cardiaque à long terme. Les auteurs 
ici ne concluent pas à un problème d'organisation des examens. Leur hypothèse est que de par les particularités de leur pathologie, symptômes négatifs et manque de conscience de la maladie, les patients n'adhèrent pas à ce type de suivi (25). En ambulatoire, les sources de difficultés sont différentes, le manque de communication et de coordination entre les soignants est souvent mis en avant pour expliquer le défaut de suivi des patients schizophrènes $(1,78,26)$. Une prise en charge par des équipes pluridisciplinaires améliore le suivi des patients (23). Outre la diffusion des bonnes pratiques, les réunions de concertation MP créent ce lien entre les équipes somatiques, psychiatriques et pharmaceutiques, indispensable à la coordination des soins.

Parmi tous les paramètres, le suivi de la pression artérielle est le résultat le moins satisfaisant de notre étude avec une diminution du PC global de suivi de $57 \%$ à $39 \%$. Sur les 2 cohortes, les informations ont été recueillies sur le dossier patient informatisé, or le suivi était meilleur en 2011. On peut supposer que la difficulté ici réside dans un défaut de traçabilité des données dans le dossier patient. II ne semble pas qu'il y ait eu un changement de pratiques pendant la période étudiée.

Le suivi métabolique des patients et en particulier le BL doivent être améliorés. Lors de l'étude de l'impact de la présence de facteurs de risque cardiovasculaire sur le suivi, les risques pris en compte ont été le diabète, les dyslipidémies, l'obésité, l'hypertension et les antécédents cardiovasculaires. Le tabac n'a pas été pris en compte, car la quasi-totalité (environ 80 \%) des détenus de la MA est fumeur. Parmi les 25 patients de la cohorte de 2015 présentant des facteurs de risques, 3 patients étaient diabétiques, 17 avaient une dyslipidémie et 8 étaient obèses. Malgré cela, il n'existe aucune différence significative dans le suivi du $B L(p=0,157)$ ou de la glycémie $(p=0,019)$, que les patients présentent des troubles métaboliques ou non. Ce défaut de suivi métabolique est souvent retrouvé dans la littérature $(18,27)$, avec notamment parmi les patients ayant initié un traitement AP moins de $20 \%$ ayant eu une mesure de leur glycémie et moins de $10 \%$ ayant eu un bilan lipidique (16). Cependant et contrairement à notre étude, le suivi était 2 à 3 fois meilleur chez les patients avec un diabète ou une dyslipidémie préexistante. Ce sont donc des examens pour lesquels médecins et pharmaciens de notre équipe doivent porter une attention toute particulière et en particulier en cas de troubles métaboliques préexistants. 
Il existe des limites à cette étude. Tout d'abord, les résultats sont difficilement comparables à ceux de la population générale. Le mode de vie en milieu fermé pourrait laisser penser que l'organisation du suivi des patients est facilitée. Or, ce milieu regroupe une population défavorisée, se souciant assez peu de son état de santé. Avant leur arrivée à la MA, leur suivi médical est bien souvent médiocre et ils sont assez peu conscients de leurs propres comorbidités. Leur faible considération pour leur état de santé et les difficultés de déplacements jusqu'à l'unité sanitaire expliquent un suivi sans doute de moins bonne qualité, que dans la population générale. Cette hypothèse est soutenue par le fait que les patients en hôpital de jour ou traités par AP retard, donc plus « accessibles » pour les équipes soignantes, semblent avoir une qualité de suivi supérieure aux autres patients.

Il est aussi nécessaire de rappeler que les recommandations sur lesquelles nous nous sommes basées pour apprécier la qualité du suivi des patients ont été adaptées au milieu pénitentiaire et diffèrent légèrement des recommandations officielles des autorités sanitaires. En effet, nous avons réduit la fréquence de suivi de tous les paramètres à un suivi annuel en l'absence d'anomalie détectée. Nous avons également écarté le périmètre abdominal des paramètres de suivi du syndrome métabolique et du risque cardiovasculaire. En effet ce critère était présent dans l'étude de 2011 mais n'a été suivi pour aucun des patients inclus. Cet examen peu onéreux et de mesure simple est considéré par les cliniciens comme trop "intrusif » par rapport à la mesure du poids. C'est cette dernière qui a été privilégiée en concertation médico-pharmaceutique.

Une autre limite de cette étude est la prise en compte des patients traités par médicaments AP à visée uniquement sédative en 2015 , alors qu'ils sont absents de la cohorte de 2011 . Ces patients présentent un faible PC du suivi paraclinique, ce qui a pu faire baisser les PC de suivi globaux pour l'année 2015 et biaiser la comparaison avec la cohorte de 2011.

Au terme de cette étude sur le suivi paraclinique des patients sous $A P$, trois axes d'améliorations pouvant optimiser la qualité du suivi ont été identifiés. Le premier de ces axes est de prioriser le suivi rapproché (au $3 e$ et au 6e mois) des patients présentant des troubles métaboliques ou un poids élevé et de ne conserver qu'un suivi annuel pour les autres patients. Lors de la première consultation, un bilan devra être systématiquement prévu pour tous les patients arrivant sous AP. Afin de faciliter cette prescription un protocole " bilan AP » a été mis à disposition sur le logiciel de prescription. Nous avons également 
décidé que pour les patients bénéficiant d'un traitement AP à visée uniquement sédative, un bilan de suivi ne sera pas systématiquement demandé considérant que ces traitements doivent être temporaires sauf en cas de coprescription de médicaments à risque de toxicité cardiovasculaire.

Ensuite, les résultats encourageants de cette étude nous permettent de proposer une nouvelle sensibilisation des prescripteurs à la toxicité métabolique des AP et en particulier, d'insister sur le suivi des patients avec un IMC supérieur à 25 , présentant des facteurs de risques cardiovasculaires ou risquant de cumuler des effets indésirables du fait de la prescription de plusieurs AP. Cette proposition s'appuie sur l'analyse des études similaires à la nôtre $(18,19,27)$ qui ont montré une amélioration du suivi des patients après sensibilisation des prescripteurs.

Enfin, une nouvelle étude sera à envisager afin de suivre l'impact de ces nouvelles propositions en réalisant notamment un focus sur les paramètres à améliorer : la PA, le suivi métabolique dans son ensemble et la mise en œuvre d'un ECG annuel.

\section{Déclaration des liens d'intérêts}

Les auteurs déclarent ne pas avoir de liens d'intérêts.

\section{BIBLIOGRAPHIE}

1. Rouillon F, Van Ganse E, Vekhoff P et al. Niveau de vigilance des psychiatres pour les facteurs de risque cardiovasculaire chez les patients schizophrènes. Encephale 2015; 41(1): 70-7.

2. Saravane D. Troubles mentaux et mortalité. Eur Psychiatry 2015; 30(8) Suppl: S7.

3. Limosin F. Schizophrénie et sujets âgés. Encephale 2009; 1 Suppl: S41-4.

4. Schürhoff F, Fond G, Berna F et al. Centres Experts Schizophrénie, un outil pour le soin et la recherche : retour sur 10 ans d'expérience. Encephale 2019; 45(1): 9-14.

5. Fève B. Effets métaboliques indésirables des antipsychotiques. Obésité 2013; 8(3): 165-73.

6. Franck N, Thibaut F. Modalités d'utilisation des neuroleptiques. EMC-psychiatr 2005; 2(4): 300-39.

7. Lefebvre N, Chéreau I, Schmitt A et al. Comorbidités somatiques chez les patients souffrants de schizophrénie traitée. Recommandations actuelles. Ann Med Psychol 2006; 164(2): 159-64. 
8. Fernandez-Egea E, Bernardo M, Donner T et al. Metabolic profile of antipsychotic - naive individuals with non-affective psychosis. Br J Psychiatry 2009; 194(5): 434-8.

9. Frimas V, Roberge C, Perroux D et al. Surveillance cardiologiques des patients traités par neuroleptiques. Encephale 2008; 34(5): 467-76.

10. Vandael E, Vandenberk B, Vandenberghe J et al. Risk factors for QTc-prolongation: systematic review of the evidence. Int J Clin Pharm 2017; 39(1): 16-25.

11. Haute Autorité de Santé. Guide-Affection de longue durée-schizophrénies. [Internet] HAS; 2007 Juin [cited 2017 Nov 22]. Available from: http://www.hassante.fr/portail/upload/docs/application/pdf/guide_ald23_schizophr_juin_07.pdf.

12. ANSM. Suivi cardio-métabolique des patients traités par antipsychotiques. [Internet] afssaps; 2010 Mars [cited 2017 Nov 22]. Available from: https://ansm.sante.fr/var/ansm_site/storage/original/application/6b3aa2489d62f9ee02e5e6 200861b2e0.pdf.

13. Saravane D, Feve B, Frances $Y$ et al. Elaboration de recommandations pour le suivi somatique des patients atteints de pathologie mentale sévère. Encephale 2009 S; 35(4): 3309.

14. FFP-CNPP. Recommandation de bonne pratique en psychiatrie : Comment améliorer la prise en charge somatique des patients ayant une pathologie psychiatrique sévère et chronique. [Internet] FFP-CNPP; 2015 Juin [cited 2019 Feb 21] Available from: http://www.psydoc-france.fr/conf\&rm/rpc/Reco_Soins_Soma_Psy.pdf

15. Juste $M$. Recommandation de bonne pratique en pharmacie clinique. Analyse d'ordonnance et niveaux d'analyse pharmaceutique. Pharm Hosp Clin 2012; 47(4): 293-5.

16. Morrato EH, Newcomer JW, Allen RR et al. Prevalence of baseline serum glucose and lipid testing in users of second-generation antipsychotic drugs: a retrospective, population-based study of Medicaid claims data. J Clin Psychiatry 2008; 69(2): 316-22.

17. Gignoux-Froment F, De Montleau F, Saravane D et al. Evaluation de la prescription d'antipsychotiques en médecine générale: conséquences métaboliques. Encéphale 2012; 38(6): 453-9.

18. Cotes RO, De Nesnera A, Kelly $M$ et al. Antipsychotic cardiometabolic side effect monitoring in a state community mental health system. Community Ment Health J 2015; 51(6): 685-94.

19. Moeller KE, Rigler SK, Mayorga A et al. Quality of monitoring for metabolic effects associated with second generation antipsychotics in patient with schizophrenia on public insurance. Schizophr Res 2011; 126: 117-23.

20. Parent G, Rose FX, Bedouch $P$ et al. Intervention pharmaceutiques émises par les pharmaciens sur les médicaments psychotropes. Encéphale 2015; 41(4): 339-45. 
21. Lee J, Dalack GW, Casher MI et al. Persistence of metabolic monitoring for psychiatry in patients treated with second-generation antipsychotics utilizing a computer-based intervention. J Clin Pharm Ther 2016; 41(2): 209-13.

22. Reeves $\mathrm{R}$, Kaldany $\mathrm{H}$, Lieberman $\mathrm{J}$ et al. Creation of a metabolic monitoring program for second generation (atypical) antipsychotics. J Correct Health Care 2009; 15(4): 292-301.

23. Coughlin $\mathrm{M}$, Goldie $\mathrm{CL}$, Tregunno $\mathrm{D}$ et al. Enhancing metabolic monitoring for children and adolescents using second-generation antipsychotics. Int J Ment Health Nurs 2018; 27(3): 1188-98.

24. Woosley RL, Heise CW, Gallo T et al. CredibleMeds: QTdrugsList. [internet] AZCERT ; 2019 Mar [cited 2019 Mar 29] https://crediblemeds.org/new-drug-list/

25. Manchia M, Firinu G, Carpiniello B et al. Clinicians' adherence to clinical practice guidelines for cardiac function monitoring during antipsychotic treatment: a retrospective report on 434 patients with severe mental illness. BMC Psychiatry 2017; 17(1): 121.

26. Jennex A, Gardner DM. Monitoring and management of metabolic risk factors in outpatients taking antipsychotic drugs: a controlled study. Can J Psychiatry 2008; 53(1): 3442.

27. Mackin P, Bishop DR, Watkinson HM. A prospective study of monitoring practices for metabolic disease in antipsychotic-treated community psychiatric patients. BMC Psychiatry 2007; 7: 28.

Figure.1 : Fiche nominative de suivi des patients sous AP élaborée en réunion de concertation médico-pharmaceutique 
Figure.1: Fiche nominative de suivi des patients sous AP élaborée en réunion de concertation médico-pharmaceutique

\section{$\underline{\text { Suivi clinique et biologique des patients sous neuroleptiques }}$}

Hospices Civils de Lyon Leveutien

Patient:.

Sexe : Fem $\square$

Masc $\square$

Date de naissance:

Taille:

Facteur de risques associés: $\quad 2$ ou+Médicaments TdP $\square$

FR CV (diabète, dyslipidémie, ATCD CV, HTA, obésité)

Date de début du traitement:

. Instauration

$\square$ Poursuite

\begin{tabular}{|c|c|c|c|c|c|c|c|c|c|c|c|c|c|c|}
\hline Données Dat & $\begin{array}{l}\text { Bilan } \\
\text { initial: }\end{array}$ & M 3: & M 6: & 1A: & 1 A M 6: & 2A: & 2AM 6: & 3A: & 3 A M 6: & 4A: & 4 A M 6: & $5 \mathrm{~A}:$ & 5 A M 6: & 6A: \\
\hline date & & & & & & & & & & & & & & \\
\hline Poids (kg) & & & & & & & & & & & & & & \\
\hline IMC (poids/taille & & & & & & & & & & & & & & \\
\hline TA & & & & & & & & & & & & & & \\
\hline Bilan lipidique & & & & & & & & & & & & & & \\
\hline Hémogramme & & & & & & & & & & & & & & \\
\hline Transaminases & & & & & & & & & & & & & & \\
\hline lonogrammesg & & & & & & & & & & & & & & \\
\hline Glycémie à jeun & & & & & & & & & & & & & & \\
\hline $\mathrm{F}^{\circ}$ rénale MDRD & & & & & & & & & & & & & & \\
\hline ECG/ FC & & & & & & & & & & & & & & \\
\hline
\end{tabular}

Commentaires: 


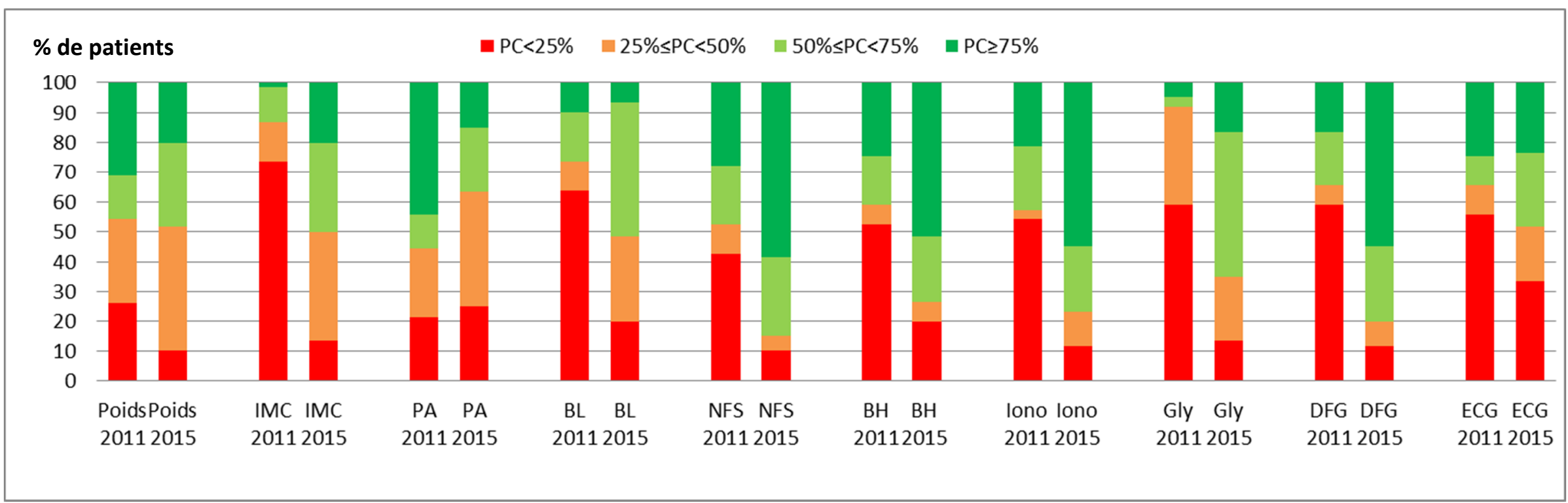

P-value : Poids (0,018), IMC $(<0,000)$, PA $(0,004), B L(<0,000)$, NFS $(<0,000)$, BH $(0,002)$, ionogramme $(<0,001)$, glycémie $(<0,000)$, DFG $(<0,000)$ et ECG $(0,03)$.

Abréviations : IMC : indice de masse corporelle ; PA : Pression artérielle ; BL : Bilan lipidique ; NFS : Numération Formule Sanguine ; BH : Bilan hépatique ; lono : lonogramme ; Gly : Glycémie ; DFG : débit de filtration glomérulaire ; ECG : électrocardiogramme. 
Tableau 1 : Pourcentages moyens de conformité (PC) pour chaque examen.

\begin{tabular}{|l|c|c|}
\hline \multirow{2}{*}{} & \multicolumn{2}{|c|}{ PC moyen (\%) } \\
\cline { 2 - 3 } & 2011 & 2015 \\
\hline Poids & 49 & 48 \\
\hline IMC & 14 & 47 \\
\hline Pression artérielle & 57 & 39 \\
\hline Bilan lipidique & 22 & 39 \\
\hline Hémogramme & 43 & 73 \\
\hline Transaminases & 36 & 65 \\
\hline lonogramme & 34 & 69 \\
\hline Glycémie à jeun & 19 & 49 \\
\hline DFG & 28 & 69 \\
\hline ECG & 33 & 42 \\
\hline
\end{tabular}

Abréviations : DFG : débit de filtration glomérulaire ; ECG : électrocardiogramme ; IMC : indice de masse corporelle 